\title{
Need to measure outcome after discharge in surgical audit
}

\author{
R H Hardwick, A Saltrese-Taylor, C D Collins
}

\begin{abstract}
Objective - To assess the accuracy of outcome data on appendicectomy routinely collected as part of a surgical audit and to investigate outcome in the non-audited period after discharge.

Design - Retrospective analysis of audit data recorded by the Medical Data Index (MDI) computer system for all patients undergoing emergency appendicectomy in one year; subsequent analysis of their hospital notes and notes held by their general practitioners for patients identified by a questionnaire who had consulted their general practitioner for a wound complication.
\end{abstract}

Setting - One district general hospital with four consultant general surgeons serving a population of 250000 .

Patients - 230 patients undergoing emergency appendicectomy during 1989.

Main measures - Comparison of postoperative complications recorded in hospital notes with those recorded by the MDI system and with those recorded by patients' general practitioners after discharge.

Results - Of the 230 patients, 29(13\%) had a postoperative complication recorded in their hospital notes, but only $14(6 \%)$ patients had these recorded by the MDI system. $189(82 \%)$ of the patients completed the outcome questionnaire after discharge. The number of wound infections as recorded by the MDI system, the hospital notes, and notes held by targeted patients' general practitioners were three $(1 \%)$, eight $(3 \%)$, and $18(8 \%)$ respectively. None of 12 readmissions with complications identified by the hospital notes were identified by the MDI system.

Conclusions - Accurate audit of postoperative complications must be extended to the period after discharge. Computerised audit systems must be able to relate readmissions to specific previous admissions.

(Quality in Health Care 1992;1:165-167)

\section{Introduction}

When conducting audit it is important to ensure that the information collected is accurate, relevant, and complete. ${ }^{1}$ This is often difficult, especially when auditing outcome, as much may happen to a patient after discharge from hospital that will not be recorded by a hospital based audit. This is particularly true of postoperative complications as patients have often left hospital before any problems have had time to develop (that is, wound infections). ${ }^{2}$

Since the beginning of 1989 the general surgeons in Taunton and Somerset Hospital have used a computerised audit system, Medical Data Index (MDI), to audit all their admissions. This system is incorporated into the patient administration system in such a way as to ensure confidentiality but to allow free flow of information from the patient administration system into MDI. Information about the admission is recorded by the house staff during the patients' stay on a card that remains in the notes until discharge, when it is removed and the information entered into the MDI database by an audit clerk and a discharge summary automatically generated. Details of the operation details are recorded on the card by the surgeon and coded according to office of Population Censuses and Surveys 4 (OPCS 4 ) at the time of the procedure.

This study was initiated after an audit report of complications after emergency appendicectomy in 1989, which suggested a wound infection rate of only $1 \cdot 3 \%$. Although $13 \%-66 \%$ of patients with infections after appendicectomy present after discharge from hospital, ${ }^{3}$ the unexpectedly low incidence of hospital infection in our series was thought to require investigation. The study set out to discover whether the predischarge audit was accurate and to assess the nature and magnitude of non-audited postoperative morbidity occurring after discharge.

\section{Patients and methods}

An audit report was generated by the MDI system detailing all 230 emergency appendicectomies performed as a primary procedure during 1989 and any associated complications. The hospital records of all these patients were then examined, and details of each admission resulting in appendicectomy were recorded. All patients were then sent a postal questionnaire with a covering letter and a prepaid envelope. This questionnaire had been piloted on 20 patients chosen at random in a routine follow up clinic to ensure that the questions were comprehensive and unambiguous. Parents of children under the age of 16 years were asked to complete the questionnaire on their child's behalf. After three weeks non-responders received a second
Accepted for publication 21 July 1992 
questionnaire with a covering letter. After a further two weeks any remaining nonresponders were contacted by telephone. ${ }^{4}$

The questionnaire asked how long it had been after discharge before the patients returned to their normal daily activities and whether they had visited their general practitioner for any reason related to their appendicectomy, and if so, why. The general practitioners of any patients who had visited their doctors with a wound complication were contacted by telephone and asked to consult their notes for details regarding the wound. Patients were ascribed to one of three groups: probable wound infection, non-specific wound pain and slight discharge, or miscellaneous. All wounds presumed to have been infected had been erythematous and discharging pus, but no bacteriological confirmation was available in most patients. All patients were treated with broad spectrum antibiotics.

\section{Results}

The notes of all 230 patients undergoing emergency appendicectomy during 1989 were available for examination. The patients had a mean age of 27 years (range 4-86 years) and a median age of 23 years. In all, 149(65\%) patients returned the questionnaire, $40(17 \%)$ completed it over the telephone, and $41(18 \%)$ patients could not be contacted. This gave an overall response rate of $82 \%$. The mean time from operation to completion of the questionnaire was 11 months (range 3-16 months).

The hospital notes disclosed 29(13\%) patients with wound complications, compared with $14(6 \%)$ identified by the MDI system. Among the 15 complications missed by the MDI system seven were wound infections, three were cases of acute urinary retention, and two were pelvic abscesses. Twelve patients were readmitted: four for wound infections, two for pelvic abscesses (one abscess resolved after antibiotic treatment and the other required drainage), two for small bowel obstruction secondary to adhesions (both settling with conservative management), and one each for incisional hernia, abdominal pain, superficial thrombophlebitis of the lower limb, and poor diabetic control. None of these readmissions were identified by the MDI system as complications of a previous admission. A review of the histological records showed that $57(25 \%)$ of the appendixes removed were non-inflamed.

Of the 189 patients who completed the questionnaire, $113(60 \%)$ visited their general practitioners after discharge. Of these, 35 were concerned that they had a wound infection, and they all visited their general practitioner. A review of the general practitioners' notes indicated that 18 patients had a probable wound infection and 17 had non-specific wound pain or a slight wound discharge. Of the 78 other patients who visited their general practitioners, 18 complained of generalised abdominal pain, two were unwell with nausea and a fever (and later found to have pelvic abscesses), one had a suspected deep vein

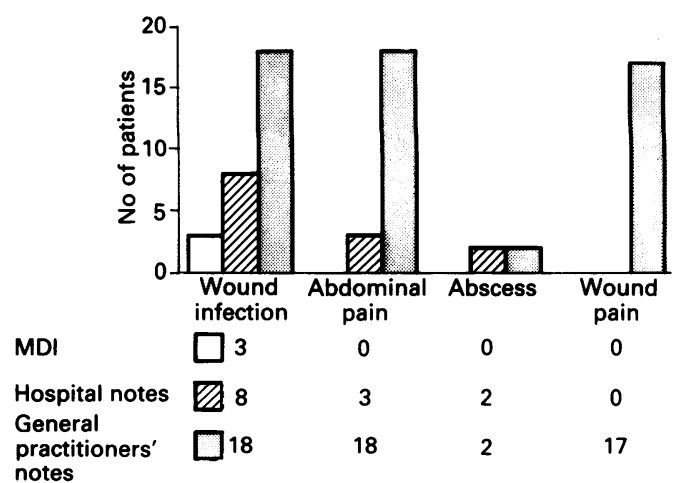

notes

Postdischarge wound complications and sepsis after appendicectomy according to three methods of audit

thrombosis, 19 had miscellaneous problems, and 38 had come for a check up, to get a certificate, or to have sutures removed. The figure compares the detection of four postoperative complications by the MDI system, the hospital notes, and the postdischarge questionnaire.

Of the patients who completed the questionnaire, 64(34\%) returned to their normal daily activities after one week but $51(27 \%)$ convalesced for longer than four weeks. There was no relation between length of convalescence and complications.

\section{Discussion}

This study shows that audit of postoperative outcome must continue after discharge, as at least $56 \%$ of the adverse postoperative events occurred after patients had returned home. After 230 appendicectomies, eight (3\%) patients had a wound infection recorded in their hospital notes, but only three $(1 \%)$ had this complication recorded by audit with the MDI system. This compares with a wound infection rate of $8 \%$ found by the patients' general practitioners, after discharge. Although incomplete note keeping may account for a small part of this difference, it seems likely that the main discrepancy is due to the inherent delay before many postoperative complications present themselves. This being the case, strategies need to be developed to audit routinely the outcome of medical intervention after discharge. ${ }^{5}$

Outpatient clinics have traditionally been used in this role, but this is an inefficient use of a scarce resource and many patients are now followed up by their general practitioner. ${ }^{6}$ As $60 \%$ of patients visited their general practitioner after surgery information on outcome after discharge could be collected by them. Alternatively, as patients have shown their willingness to return questionnaires about recent hospital admissions, a routine questionnaire survey might be feasible, so that all patients report back to the hospital about their own outcome. Whichever strategy is developed, however, collection of data on outcome needs to be coordinated, with input from primary and secondary health care practitioners and a free flow of information between these two groups, if meaningful measurements of outcome are to be obtained. ${ }^{7}$ 
Much of the underreporting of complications in the audit with the MDI system was found to be due to the inability of the system to relate readmission with a complication to the relevant previous admission. This has now been corrected. The accuracy and completeness of the MDI data depend on the enthusiasm of the doctors filling out the cards, and a regular "quality control" check of the data being entered into the system is important. ${ }^{8}$

Notably, nearly a third of patients had still not resumed their normal daily activities after four weeks at home, but the reasons for this are not known. There was no apparent relation with complications so the personality of the patient and the advice given by his or her doctor may be important factors in determining how quickly a patient returns to normal activities. A study to investigate this would be of interest, as little is known about the relative importance given by patients to postoperative advice from the hospital staff compared with that received from their general practitioners.

In summary, audit of outcome of surgery must extend into the period after discharge if it is to have any real meaning, and the accuracy of all audit data should be periodically checked. Improved flow of information between general practitioners and hospital doctors, plus the use of routine patient questionnaires after discharge may provide the most efficient answer to the problem of extending audit into the community.

1 Devlin HB. Audit and the quality of clinical care. Ann R Coll Surg Engl 1990, suppl.

2 Law JW, Fadel Misfuriki S, Jeffery PJ. The importance of surveillence after discharge from hospital in the diagnosis of postoperative wound infection. Ann $R$ Coll Surg Engl 1990;72:207-9.

3 Krukowski ZH, Irwin ST, Denholm S, Matheson NA. Preventing wound infection after appendicectomy: a review. Br f Surg 1988;75:1023-33.

4 Dillman DA Mail and telephone surveys: the total design method. New York: Wiley Interscience Publications, method.

5 Macintyre IMC. Extending surgical audit: the assessment of postoperative outcome. Br f Surg 1989;76:531-2.

6 McCormack TT, Collier JA, Able PD, Collins CD, Ritchie WN. Attitudes to follow-up after uncomplicated surgery - hospital outpatients or general practitioner? Health Trends 1984;16:46-7.

7 Frater A. Health outcomes: a challenge to the status quo Quality in Health Care 1992;1:87-8.

8 Gruer R, Gordon DS, Gunn AA, Ruckley CV. Auditing surgical audit. Lancet $1986 ; \mathrm{i}: 23-6$. 